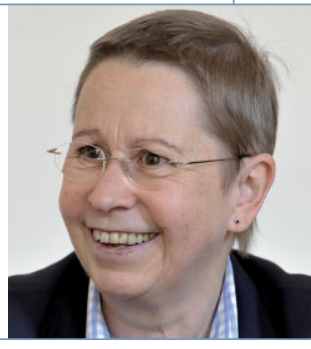

Ulrike Beisiegel

\title{
Vorschläge für eine Entschleunigung in der Wissenschaft
}

Die Diskussion des 4. Berliner Wissenschaftsgespräch der Robert Bosch Stiftung im Herbst 2009 hat sich mit der Leistungsfähigkeit der Wissenschaft in einer Zeit des extremen Wissenszuwachses und eines hohen gesellschaftlichen Anspruchs an die Wissenschaft beschäftigt. Die Teilnehmer des Gesprächs haben ein Thesenpapier zusammengestellt, das die wichtigsten Probleme im heutigen Wissenschaftssystem aufgreift. Die sieben Thesen beschreiben Entschleunigungsstrategien, mit denen wieder mehr Raum und Zeit für kritische Reflexionen ermöglicht werden soll.

Fakt und zentrale Herausforderung ist die Tatsache des zunehmenden Wissens und der damit einhergehenden wachsenden Komplexität. Um in dieser Situation die Qualität der Forschung und damit die Relevanz der Forschungsergebnisse zu erhalten, müssen wir nicht immer mehr publizieren, sondern besonders sorgfältig prüfen, wie sich neue Ergebnisse in das bestehende Wissensnetz einfügen. Es kann nicht sein, dass die Zahl der Publikationen als Evaluationskriterium für wissenschaftliche Leistungen herangezogen wird. Vielmehr müssen die Ergebnisse in Bezug auf ihren Beitrag zum Wissensgewinn beurteilt werden.

Die Erarbeitung von neuem Wissen, d. h. die Erstellung eines soliden Forschungskonzeptes, die sorgfältige Durchführung von gerade in den Biowissenschaften - komplexen Experimenten, die kritische Bewertung der Ergebnisse und ggf. die Korrektur unvermeidlicher Irrtümer im Versuchsaufbau sowie die Reflexion der Bedeutung der Ergebnisse benötigt Zeit und Ressourcen. Letztere werden in dem Thesenpapier nachdrücklich auch als verlässliche Grundfinanzierung der Forschung im Sinne der Allgemeinheit eingefordert. Die „Forschung für den Menschen“ soll auch von den Menschen unterstützt werden. Sie muss jedoch substanziell und nachvollziehbar sein. Wir müssen uns an den
Inhalten unserer Forschung und der Qualität der Forschungsergebnisse messen lassen. Und es darf nicht sein, dass es, wie Wolfgang Frühwald es 2010 beschrieb, „in Projekten nicht mehr um die Substanz des neuen Wissen, sondern nur noch um dessen Sichtbarkeit“ geht. Er spricht in diesem Zusammenhang von einer „Bluffgesellschaft“.

Warum müssen Wissenschaftler jedoch überhaupt „bluffen“? Die Antwort liegt im Grunde darin, dass wir auf der Basis von vielversprechenden Technologieentwicklungen eine rasante Ökonomisierung der Wissenschaft erleben, die zu einem exzessiven Wettbewerb im globalen Wissenschaftssystem führen und wir uns heute auf „Forschungsmärkten“ behaupten müssen. Autorschaften sind zur Währung der Wissenschaft geworden und bedingen die Mittelzuweisungen. Autorschaften auf Publikationen dokumentieren so oft nicht mehr den substanziellen Beitrag der entsprechenden Person sondern werden strategisch genutzt. Der Druck im Wettbewerb Forschungsmittel einzuwerben ist extrem gewachsen und Anträge basieren oft nicht mehr auf wissenschaftlichen Vorarbeiten und dadurch erstellten Konzepten und Hypothesen, sondern es hat sich ein professionelles Antragswesen gebildet für das Agenturen standardisierte Anträge erstellen.

Ein Ausgangspunkt der Diskussion war die Definition der Forschung als „der Wahrheit verpflichteter Erkenntnisgewinn“ und damit einem Prozess, der „unter der Autorität des Zweifels“ stehen muss und Zeit für entsprechende Reflexion und Korrekturen benötigt. Gerade in den Biowissenschaften erleben wir heute eine extrem schnelle Methodenentwicklung und oft lässt die Faszination des Möglichen für diese Reflexion keine Zeit. Es wird zu wenig nachgedacht über die Frage, wie sich neue Technologien auf den Menschen, seine sozialen Lebensformen und die ihn umgebende Natur auswirken können. Die
Wissenschaft kann so leicht zum Selbstzweck werden, bei dem nicht mehr die Wahrheitssuche im Vordergrund steht sondern die Faszination der Technik. Technik, die aus anderen Fachdisziplinen kommt und die der Wissenschaftler nicht ohne weiteres in ihren Grundlagen nachvollziehen kann. Gerade dann müssen die experimentellen Grundlagen jedoch transparent gemacht werden damit die Datenqualität nachvollziehbar und überprüfbar ist. Dies ist bei vielen Publikationen nicht mehr der Fall.

Fehlt der Wahrheitsanspruch in der Forschung und tritt die gesellschaftliche Relevanz in den Hintergrund, überwiegt das abstrakte Zukunftspotential als Ziel und der Konkurrenzkampf um dieses Potential und verführt zu Unredlichkeiten und wissenschaftlichem Fehlverhalten. Diese Erfahrung hat die DFG in ihrer Initiative „Qualität statt Quantität“ aufgegriffen und sie sollte dazu bewegen, uns die Zeit zur kritischen Diskussion zu nehmen um die Qualität der Forschung langfristig zu sichern. Ich bin sicher, dass nur Ehrlichkeit im Umgang mit Daten und Integrität zu wissenschaftlichem Fortschritt für unsere Gesellschaft führt.

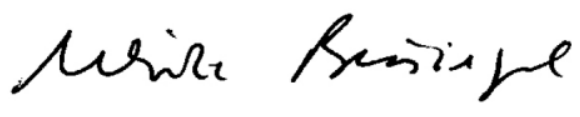

Ulrike Beisiegel,

Präsidentin der Georg-August-Universität Göttingen

Korrespondenzadresse:

Prof. Dr. Ulrike Beisiegel

Georg-August-Universität Göttingen

Wilhelmsplatz 1

D-37073 Göttingen

Tel.: 0551-39-4311

Fax : 0551-39-4135

praesidentin@uni-goettingen.de

www.bosch-stiftung.de/content/language 1/

downloads/WissenGespraech_11_09_Protokoll

entwurf.pdf 